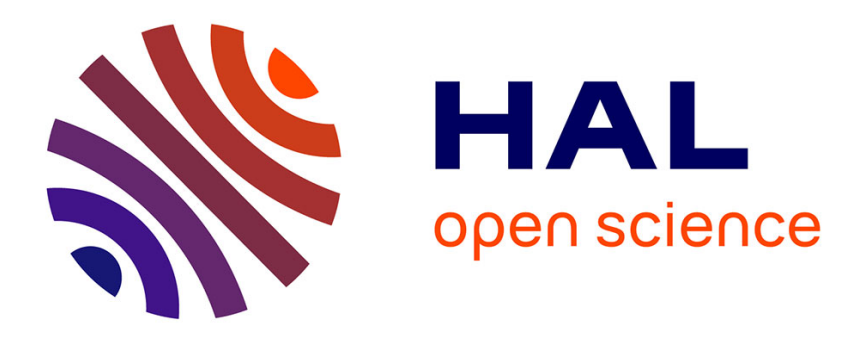

\title{
A Model of Material with Phase Change and Applications
}

Z. Moumni, Q. Nguyen

\section{To cite this version:}

Z. Moumni, Q. Nguyen. A Model of Material with Phase Change and Applications. Journal de Physique IV Proceedings, 1996, 06 (C1), pp.C1-335-C1-345. 10.1051/jp4:1996132 . jpa-00254164

\section{HAL Id: jpa-00254164 https://hal.science/jpa-00254164}

Submitted on 1 Jan 1996

HAL is a multi-disciplinary open access archive for the deposit and dissemination of scientific research documents, whether they are published or not. The documents may come from teaching and research institutions in France or abroad, or from public or private research centers.
L'archive ouverte pluridisciplinaire HAL, est destinée au dépôt et à la diffusion de documents scientifiques de niveau recherche, publiés ou non, émanant des établissements d'enseignement et de recherche français ou étrangers, des laboratoires publics ou privés. 


\title{
A Model of Material with Phase Change and Applications
}

\author{
Z. Moumni and Q.S. Nguyen \\ Laboratoire de Mécanique des Solides, Centre Commun X-Mines-Ponts-CNRS, URA $n^{\circ} 317$, Ecole \\ Polytechnique, 91128 Palaiseau cedex, France
}

\begin{abstract}
The macroscopic model of solid-solid phase change presented makes use of internal variables to represent the phase fractions and the local strains. As such, it can be cast in the framework of standard materials with internal constraints. These constraints are accounted for by Lagrange's multipliers. Reversible or irreversible phase changes are considered in a general way. Material stability is also discussed. Two examples are provided to illustrate the application of the proposed model to brittle damage and to shape memory materials. This model enable to solve boundary value problems as it is shown for the anti-plane shear crack one.
\end{abstract}

\section{Introduction}

In this paper, a simple material model of phase change is presented and applied to the constitutive modelling of shape memory alloys and to the description of the propagation of damage in a brittle structure. Phase change is described by an internal variable which represents the transformed fraction. The considered model enters the framework of internal variable description with internal constraints in view of the definition of the internal variable and its dependence on the macroscopic strain.

Constitutive models with internal constraints have been considered in a general way by Frémond [1], using systematically the formalism of sub-differential introduced by Moreau [2]. Frémond's work could be seen as an extension of the generalized standard formalism, eg. Germain [3].

The constitutive equations for standard materials with internal constraints are presented in the second section of this paper. In the third section, the model is introduced as a simple example of standard materials with internal constraints. Reversible and irreversible phase changes are then discussed in a general way and material stability is examined. In the fourth section, attention is focussed on the description of the extension of damage zone in a solid with a model of partial and brittle damage. The last section deals with the constitutive description of shape memory alloys following the lines of Frémond [1] and Müller [5]. In particular, the formation of hysteresis loops observed in stress-strain curves is discussed.

\section{Standard materials with internal constraints}

Consider a material defined by the collection of state variables $(\epsilon, \beta, \alpha, \mathrm{T})$ which are, the strain tensor, the reversible and irreversible internal parameters and the temperature respectively. For the sake of simplicity, the assumption of small transformation is admitted although it is not necessary. The free energy per unit volume is function of the state variables:

$$
W=W(\epsilon, \beta, \alpha, T) .
$$

The state variables dependance is expressed by $M$ equality constraints: $g_{m}(\epsilon, \beta, \alpha)=0$, with $m=$ 1 to $M$ and by $N$ inequality constraints : $h_{n}(\epsilon, \beta, \alpha) \geq 0$, with $n=1$ to $N$.

The rates $\dot{\epsilon}, \dot{\beta}, \dot{\alpha}$ are thus not arbitrary, but must be compatible with the constraints :

$$
\frac{\partial g_{m}}{\partial \epsilon} \dot{\epsilon}+\frac{\partial g_{m}}{\partial \beta} \dot{\beta}+\frac{\partial g_{m}}{\partial \alpha} \dot{\alpha}=0 \text { and } \frac{\partial h_{n}}{\partial \epsilon} \dot{\epsilon}+\frac{\partial h_{n}}{\partial \beta} \dot{\beta}+\frac{\partial h_{n}}{\partial \alpha} \dot{\alpha} \geq 0 \text { if } h_{n}=0 .
$$


The entropy density $s$ is given by the classical relation : $s=-\frac{\partial W}{\partial T}$. The intrinsic dissipation per unit volume is, eg. Germain [3] :

$$
d=\sigma \dot{\epsilon}-\frac{\partial W}{\partial \epsilon} \dot{\epsilon}-\frac{\partial W}{\partial \beta} \dot{\beta}-\frac{\partial W}{\partial \alpha} \dot{\alpha} \geq 0
$$

in which, the partial derivatives of energy $W$ with respect to its arguments $\epsilon, \beta$ and $\alpha$ are the generalized forces. It is assumed that all constraints are perfect in the sense that all generalized reactions are associated with the potential :

$W_{c}=-\lambda_{m} g_{m}-\mu_{n} h_{n}$ with $\mu_{n} \geq 0$ and $\mu_{n} h_{n}=0,\left(\lambda_{m}\right.$ and $\mu_{n}$ are the Lagrange's multipliers. $)$

It follows from (1) and (4) that the material has the potential energy $L=W+W_{c}$ which is the associated Lagrangean. The associated generalized forces are :

$$
\sigma_{r}=\frac{\partial L}{\partial \epsilon},-B=\frac{\partial L}{\partial \beta} \text { and }-A=\frac{\partial L}{\partial \alpha} .
$$

The assumption of perfect constraints implies the following relations (obtained by time differentiation of equalities $\lambda_{m} g_{m}=0$ and $\mu_{n} h_{n}=0$ ) :

$$
\begin{gathered}
\lambda_{m} \frac{\partial g_{m}}{\partial \epsilon} \dot{\epsilon}+\lambda_{m} \frac{\partial g_{m}}{\partial \beta} \dot{\beta}+\lambda_{m} \frac{\partial g_{m}}{\partial \alpha} \dot{\alpha}=0 \\
\mu_{n} \frac{\partial h_{n}}{\partial \epsilon} \dot{\epsilon}+\mu_{n} \frac{\partial h_{n}}{\partial \beta} \dot{\beta}+\mu_{n} \frac{\partial h_{n}}{\partial \alpha} \dot{\alpha}=0 .
\end{gathered}
$$

Consequently, generalized reactions never work in the actual evolution.

It follows from (6) and (7) that the dissipation can also be written as :

$$
d=\left(\sigma-\sigma_{r}\right) \dot{\epsilon}+B \dot{\beta}+A \dot{\alpha} \geq 0 .
$$

Coleman's argument is now applied to this expression of the dissipation as if all variables were independent : by definition, $\beta$ are reversible variables, then the associated force $B$ must be zero. The same conclusion is available for all reversible variables. For example, if the deformation itseft is not an irreversible mechanism, then : $\sigma=\sigma_{r}$. This means that irreversible stress does not exist in the considered model. This assumption will be maintained in what follows to simplify the presentation. The expression of dissipation simplifies to read :

$$
d=A \dot{\alpha}
$$

where $A$ is defined in (5).

The set of equations (5) must be completed by the evolution equation of irreversible variables. Standard laws of evolution relies on the existence of a dissipation potential $D$, which is a function of the rate $\dot{\alpha}$ and eventually may depend on the present state via the current values of the state variables, such that:

$$
A=\frac{\partial D}{\partial \dot{\alpha}}
$$

If the behaviour is time-independent as in incremental plasticity, $D$ is then a positive, homogeneous function of degree one.

Equations (5) and (10) defines completely the reversible and irreversible behaviour of generalized standard models with internal constraints. The two presentations, by Lagrange formalism or by Moreau formalism, appear to be stricly equivalent in essence. 


\section{A simple model with phase change}

\subsection{A model of material}

In this section, a simple model of solids with phase change is presented as an example of standard materials with internal constraints. The phase transformation, depending on the physical context of the problem, may be reversible or irreversible. For example, in the modelling of shape memory materials, the martensite-austenite transformation can be considered as a reversible one since the observed dissipation is rather small. In the description of damage of solids, damaged and undamaged materials may be considered as two different phases and the extension of damage inside a solid is then an irreversible phase transformation.

The phase transformation between two phases is described by the internal parameter $z$ which is the local transformed fraction.

The considered model, as shown in fig.(1), can be represented by a series arrangement of springs with state variables $\epsilon, e, f, z$ where $\epsilon$ denotes the macroscopic strain tensor, while $e$ and $f$ are local strain tensors of each phase and $z$, the transformed fraction.

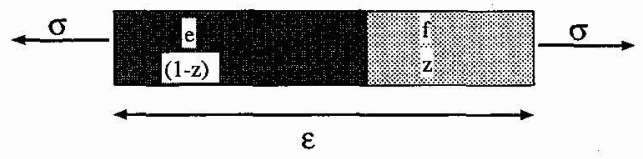

Figure 1: onedimensional model.

These variables are related by perfect internal constraints :

$$
(1-z) e+z f-\epsilon=0, z \geq 0, \text { and } 1-z \geq 0 .
$$

For the sake of simplicity the temperature is kept constant and to begin with, only isothermal transformations are considered. The free energy consists of the stored energy in each phase $U$ and $V$ and, eventually, of a phase interaction energy $I(z)$ :

$$
W=(1-z) U(e)+z V(f)+I(z) .
$$

The phase interaction energy must naturally satisfy the condition : $I(0)=I(1)=0$.

The associated Lagrangean is :

$$
L=(1-z) U(e)+z V(f)+I(z)-\lambda((1-z) e+z f-\epsilon)-\lambda_{1} z-\lambda_{2}(1-z) .
$$

The reversible variables $\beta$ are $e, f$ and $z$ if local deformations and phase change are reversible processes. The state equations are then :

$$
\frac{\partial L}{\partial \epsilon}=\sigma, \quad \frac{\partial L}{\partial e}=0, \quad \frac{\partial L}{\partial f}=0, \quad \frac{\partial L}{\partial z}=0,
$$

with additional relations related to the perfect constraints :

$$
\lambda_{1} \geq 0, \quad \lambda_{2} \geq 0 \quad \text { with } \quad \lambda_{1} z=0, \quad \lambda_{2}(1-z)=0 \text { and } \quad \lambda((1-z) e+z f-\epsilon)=0 .
$$

It is important to note that the relations (11) to (15) are sufficient to obtain the unknowns $\sigma, e, f, z, \lambda, \lambda_{1}$ and $\lambda_{2}$ in terms of $\epsilon$. These equations are explicitely written as :

$$
\begin{gathered}
\sigma=\lambda, \quad(1-z)\left(\lambda-U_{, e}\right)=0, \quad z\left(\lambda-V_{, f}\right)=0 \\
V(f)-U(e)+(f-e) \lambda+\lambda_{2}-\lambda_{1}+I^{\prime}(z)=0
\end{gathered}
$$


For example, if $0<z<1$ then :

$$
\sigma=\lambda=U_{, e}=V, f
$$

Hence, the reversible case leads to a nonlinear elastic material.

If the phase change is irreversible while local deformations are reversible, then $\beta=(e, f)$ and $\alpha=z$. Then, state equations are :

$$
\frac{\partial L}{\partial \epsilon}=\sigma, \quad \frac{\partial L}{\partial e}=0, \quad \frac{\partial L}{\partial f}=0, \quad \frac{\partial L}{\partial z}=-A .
$$

In this case, it is necessary to complete the description by the equation of evolution of the transformed fraction. Since $z$ is a scalar, this equation can always be written as (10) with a dissipation potential $D$ function of $\dot{z}$ and of the present state $(\epsilon, e, f, z)$. For time-independent behaviour, $D$ must be a positive homogeneous function of $\dot{z}$ and it is well known as in incremental plasticity that equation (10) can also be written in the form of the normality law :

$$
\dot{z}=\nu \frac{\partial f}{\partial A} \quad \text { with } \quad f=f(A, \epsilon, e, f, z) \leq 0 \quad \text { and } \quad \nu f=0 .
$$

In (17), the inequality expresses the present domain of admissible forces in the same spirit as a criterion of plasticity.

Since $A$ is a number, the assumption of a convex domain of admissible forces leads to :

$$
A^{-}(\epsilon, e, f, z) \leq A \leq A^{+}(\epsilon, e, f, z)
$$

where $A^{+}>0$ and $A^{-}<0$ denote respectively the upper and lower limits of the admissible forces.

The associated normality law is :

$$
\dot{z}=0 \text { if } A^{-}<A<A^{+}, \dot{z} \leq 0 \text { if } A=A^{-}, \dot{z} \geq 0 \text { if } A=A^{+}
$$

It is important to note that, since $A$ may be multivalued, these equations must be understood in the following sense. All the set of values of $A$ must belong to the interval $\left[A^{-}, A^{+}\right]$. If there exists a particular value $A$ equal to $A^{-}$then $\dot{z} \leq 0$. If there exists a particular value $A$ equal to $A^{+}$then $\dot{z} \geq 0$. If all values of $A$ are strictly inside this interval defined by the two extremities $A^{-}$and $A^{+}$, then $\dot{z}$ is equal to zero. The fact that Lagrange multipliers are not uniquely defined theoretically induces no difficulty.

The associated dissipation potential is :

$$
\begin{aligned}
& D=A^{-}(\epsilon, e, f, z) \dot{z} \text { if } \dot{z}<0, \\
& D=A^{+}(\epsilon, e, f, z) \dot{z} \text { if } \dot{z}>0 .
\end{aligned}
$$

Constitutive models with phase change involving more than two phases can be also introduced without difficulty following the same line of reasonning.

\subsection{Material stability}

The stability of the material model under controlled stresses is now investigated. This discussion follows directly from general results established in the classical contexts of elasticity [6] and of incremental plasticity [8].

If phase change is reversible, the model obtained is one of nonlinear elasticity. It is known that for an elastic material of energy density $W(\epsilon)$, the material stability under applied stresses is obtained from the criterion of second variation :

$$
\delta^{2} W=\delta \epsilon . W,,_{\epsilon \epsilon}, \delta \epsilon>0 .
$$


Here, this criterion is difficult to apply since the explicit expression of the energy $W(\epsilon)$ is unknown. This problem has been discussed however in references [6],[8] and appears as a particular case of the stability of an elastic structure under perfect constraints. It has been shown that in this case, the stability of the material under force control is ensured if the second variation of the Lagrangean is always positive :

$$
\delta^{2} L=\delta a . L,_{a a} . \delta a>0
$$

on the set of admissible rates $\delta a$, in which $a=(\epsilon, e, f, z)$ denotes the collection of state variables. Admissible rates must satisfy :

$$
\begin{gathered}
\text { if } z=0: \delta \epsilon, \delta e, \delta f \text { are arbitrary, } \delta z \geq 0 \\
\text { if } 0<z<1:(f-e) \delta z+z \delta f+(1-z) \delta e=\delta \epsilon
\end{gathered}
$$

Thus, if $0<z<1$, the second variation of the Lagrangean is :

$$
(1-z) \delta e . U_{, e e} . \delta e+z \delta f . V_{, f f} \delta f+I^{\prime \prime}(z) \delta z^{2}>0
$$

and it is strictly positive on the set of admissible rates if and only if $U$ and $V$ are positive-definite and $I^{\prime \prime}(z)>0$. The existence of convex energy densities $U(e), V(f)$ and of a convex interaction energy $I(z)$ thus ensures the stability of the material under controlled stresses.

If phase change is irreversible, the material-stability analysis follows from the general results which have been established for stability and bifurcation analysis of dissipative systems [8].

For a system with internal constraints, the general criterion of stability can be written as :

$$
\delta a . L,_{a a} . \delta a+\delta a . D,_{a} . \delta z>0
$$

for all admissible rates.

For example, if $A=A^{+}, 0<z<1$ and if $A^{+}$depends only on the present value of $z$ then the criterion of stability is :

$$
(1-z) \delta e . U_{, e e} \delta e+z \delta f . V,_{f f} . \delta f+\left(I^{\prime \prime}(z)+A^{+^{\prime}}(z)\right) \delta z^{2}>0
$$

for all rates satisfying: $(f-e) \delta z+(1-z) \delta e+z \delta f=\delta \epsilon$ and $\delta z \geq 0$.

It is then clear that material stability is ensured for force control if $I^{\prime \prime}(z)+A^{+^{\prime}}(z)>0$ and if the energy functions $U$ and $V$ are strictly convex.

In this case, the presence of a increasing upper limit $A^{+}$with $z$ may be sufficient to ensure the material stability even if $I(z)=0$.

\section{Applications to brittle and progressive damage}

\subsection{Constitutive model}

The constitutive modelling of brittle and progressive damage has been discussed by many authors, eg. Francfort and Marigo [9]. As shown in fig.(2), we are interested by a model describing the lost of stiffness of a material from its initial undamaged state in a progressive and time-independent manner into a residual elastic state. This model may be usefull to explain the loss of stiffness due to the extension of local damage sush as micro-cracking or the partial debonding between fiber and matrix or in multilayered composites.

Marigo and Franckfort have introduced their model to describe the problem of partial and brittle damage in a solid. Their parameter $z$ is a damage variable representing the volumic fraction of the final state. Damage appears as a mixture of the initial and the final states at the macroscale. 


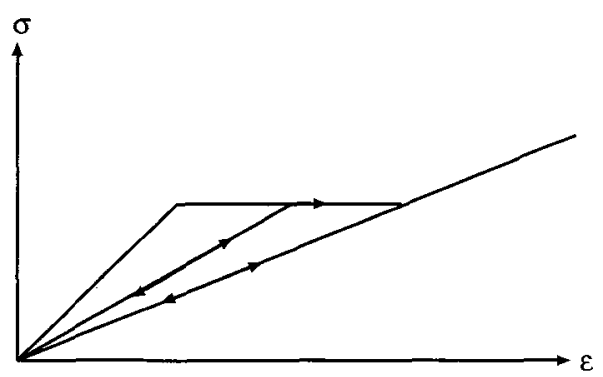

Figure 2: Progressive and partial damage.

Following their ideas, a model of progressive and brittle damage is now considered to discuss a complementing aspect of the proposed model. This discussion is different from Marigo and Franckfort's one since an evolution law of damage is now introduced in the spirit of (17) for irreversible damage. This leads to a natural model of progressive damage in the sense of incremental plasticity. A model of nonlinear elasticity is also naturally associated with equations (14), when damage may be considered as reversible and leads to an explicit value of $z$ in terms of the macrostrain $\epsilon$. For simplicity, the one dimensional case is first considered.

With the following choice :

$$
I(z)=0, \quad U(e)=\frac{1}{2} K_{1} e^{2}, \quad V(f)=\frac{1}{2} K_{2} f^{2}+k \quad \text { with } \quad r=\frac{K_{1}}{K_{2}}>1
$$

equations (13) and (14) lead to this a particular model of nonlinear elasticity :

$$
\begin{array}{r}
\text { if }|\epsilon| \leq E \text { then } z=0 \text { and } \sigma=K_{1} \epsilon \\
\text { if } E \leq|\epsilon| \leq F \quad \text { then } z=\frac{|\epsilon|-E}{F-E} \text { and } \sigma=K_{1} E \operatorname{sign}(\epsilon) \\
\text { if }|\epsilon| \geq F \text { then } z=1 \text { and } \sigma=K_{2} \epsilon
\end{array}
$$

with :

$$
E=\sqrt{\frac{2 k}{K_{1}(r-1)}}, \quad F=r E .
$$

The associated energy density is obtained from $U$ and $V$ by convexification as shown in fig.(3). The constant $k$ represents here the (reversible) phase change energy.

An irreversible model can be introduced with the following modifications :

$$
V(f)=\frac{1}{2} K_{2} f^{2}, A \neq 0, A^{+}=k, A^{-}=-\infty
$$

The dissipation is then $D=k \dot{z}$, the coefficient $k$ representing the energy dissipated by damage per unit volume.

In the three-dimensions case, the following laws of isotropic damage can be introduced as an extension of the one dimensional case. The energy functions are :

$$
U(e)=\frac{1}{2} K_{1}\left(e_{i i}\right)^{2}+\mu_{1} e_{i j}^{\prime} e_{i j}^{\prime}, \quad V(f)=\frac{1}{2} K_{2}\left(f_{i i}\right)^{2}+\mu_{2} f_{i j}^{\prime} f_{i j}^{\prime}+\delta k
$$



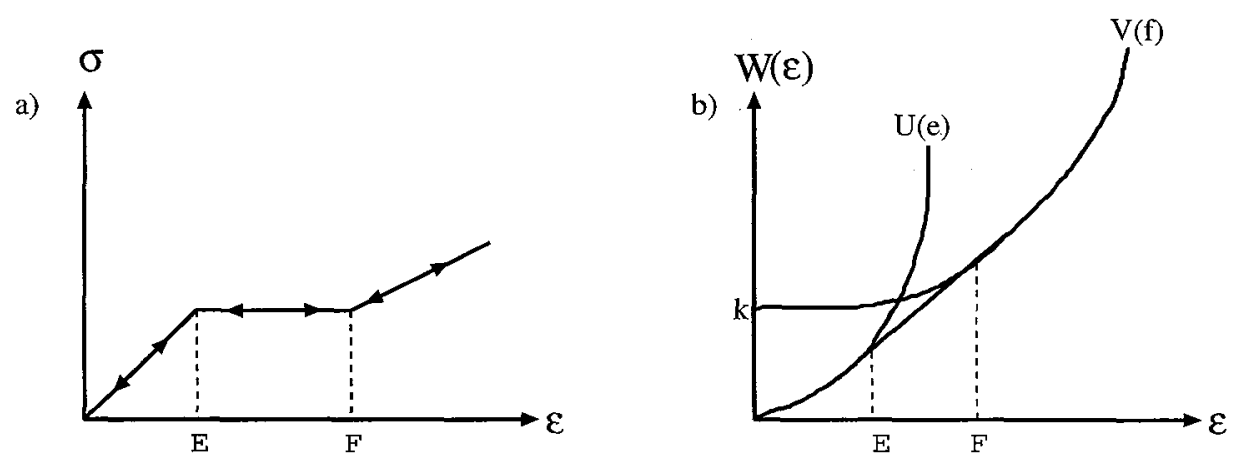

Figure 3: Convexification of the energie, $a$ : stress versus strain, $b$ : associated energie.

where $K_{1}>K_{2}$ and $\mu_{1}>\mu_{2}$ denote respectively various bulk and shear modulus. The Parameter $\delta$ takes for value 1 if the behaviour is reversible and zero if irreversible.

With the following notations :

$$
\begin{gathered}
r^{\prime}=\frac{\mu_{1}}{\mu_{2}}, E^{\prime}=\sqrt{\frac{k}{\mu_{1}\left(r^{\prime}-1\right)}}, \quad F^{\prime}=r^{\prime} E^{\prime} \\
\left|\epsilon^{\prime}\right|=\sqrt{\epsilon_{i j}^{\prime} \epsilon_{i j}^{\prime}} \quad, \quad p=\frac{1}{3} \sigma_{i i} \quad, \quad \theta=\epsilon_{i i}
\end{gathered}
$$

the results obtained in the reversible case are :

$$
\begin{aligned}
& \text { if } X=\frac{\left|\epsilon^{\prime}\right|^{2}}{E^{\prime 2}}+\frac{\theta^{2}}{E^{2}}-1<0 \quad \text { then } z=0, \quad p=K_{1} \theta \quad, \quad \sigma^{\prime}=2 \mu_{1} \epsilon^{\prime} \text {. } \\
& \text { if } Y=\frac{\left|\epsilon^{\prime}\right|^{2}}{F^{\prime 2}}+\frac{\theta^{2}}{F^{2}}-1>0 \quad \text { then } z=1, p=K_{2} \theta \quad, \quad \sigma^{\prime}=2 \mu_{2} \epsilon^{\prime} \text {. } \\
& \text { If } X>0, Y<0 \text { then }
\end{aligned}
$$

$z$ is the unique solution in the interval $[0,1]$ of equation

$$
\frac{\left|\epsilon^{\prime}\right|^{2}}{E^{\prime 2}\left(1+\left(r^{\prime}-1\right) z\right)^{2}}+\frac{\theta^{2}}{E^{2}(1+(r-1) z)^{2}}=1
$$

and

$$
p=\frac{K_{1}}{z r+(1-z)} \theta \quad, \quad \sigma^{\prime}=\frac{2 \mu_{1}}{z r^{\prime}+(1-z)} \epsilon^{\prime}
$$

The fact that equation (23) admits only one solution in the interval $[0,1]$ can be seen geometrically since it can be represented by a family of ellipses in the plane $(x, y), \quad x=\frac{\left|\epsilon^{\prime}\right|}{E^{\prime}}$ and $y=\frac{\theta}{E}$ of principal axes $(1+(r-1) z)$ and $\left(1+\left(r^{\prime}-1\right) z\right)$ when $z$ varies. A geometrical point of coordinates $(x, y)$ of the region limited by the two extreme ellipses (obtained when $z=0$ and $z=1$ ) belongs to one and only one ellipse of this family since the domain interior to each ellipse is increasing in size and contains the previous one when $z$ increases.

The three-dimensional behaviour is thus given explicitly and the constitutive equations are mathematically consistent if $r>1, r^{\prime}>1$.

If $r=r^{\prime}$ or $r=1$, or if $r^{\prime}=1$, the model is a straightforward extension of the one-dimensional case [10].

The irreversible case, with $\delta=0$ and $D=k \dot{z}$ and $\dot{z} \geq 0$, leads to an incremental behaviour in a similar way as in incremental plasticity. The non-decreasing parameter $z$ can be compared to the plastic strain. 


\subsection{Illustration : mode III fracture.}

In this subsection, we examine the problem of a crack under anti-plane shear conditions. The material of the structure undergoes reversible phase change. To simplify we assume that $(r=1)$. An exact solution is found through the hodograph method.

\subsubsection{Governing equations}

The crack is in the plane $x_{2}=0$ with its tip coinciding with the origin of the reference. The polar coordinates at the crack tip cf. figure $(4)$ are $(r, \theta)$.

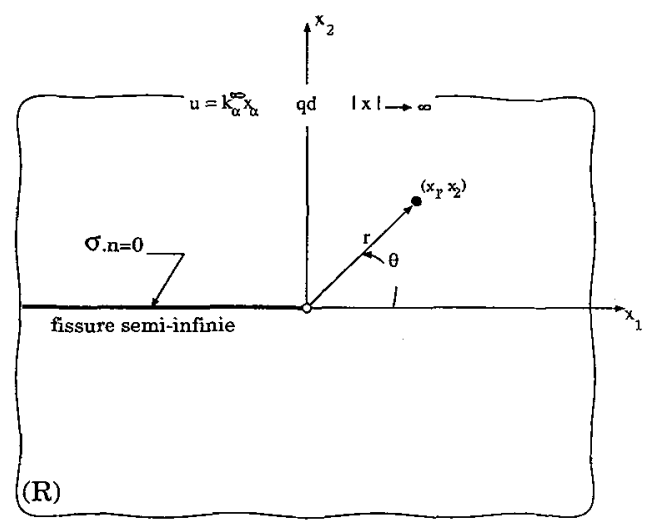

Figure 4: Anti-plane crack problem.

Our attention is focussed in an anti-plane mode. This means that the displacement satisfies :

$$
u_{\alpha}=0, \text { for } \alpha=1,2 \text { and } u_{3}=u=k^{\infty} x_{2} \text { if } x_{1}^{2}+x_{2}^{2} \text { tends to }+\infty
$$

$k^{\infty}$ is the amplitude of the shear at infinity.

Then the problem consists on finding a solution $u, z(u)$ that satisfies :

$$
\begin{aligned}
& \left(\mu(|\nabla u|) u,_{\alpha}\right),_{\alpha}=0 \quad \forall\left(x_{1}, x_{2}\right) \quad \in \Re . \\
& u, 2\left(x_{1}, x_{2}= \pm 0\right)=0 \quad \forall \quad x_{1}<0 \\
& r \rightarrow \infty \quad\left\{\begin{array}{l}
u \approx \frac{K_{U L}}{\mu_{1}} \sqrt{\frac{2 r}{\pi}} \sin \left(\frac{\theta}{2}\right) \\
u_{, x_{1}} \approx-\frac{K_{H U}}{\mu_{1}}(2 \pi r)^{-1 / 2} \sin \left(\frac{\theta}{2}\right) \\
u_{, x_{2}} \approx \frac{K_{L I}}{\mu_{1}}(2 \pi r)^{-1 / 2} \cos \left(\frac{\theta}{2}\right)
\end{array}\right.
\end{aligned}
$$

$K_{I I I}$ is the stress-intensity factor in the case of a crack in an anti-plane mode for an elastic material whose mechanical caracteristics are those of the initial phase.

\subsubsection{Solution}

A formal solution to this non-linear problem is obtained by the use of the hodograph transformation in which the first derivatives of $u$ are introduced as the independant variables. The details of this analysis is found in [10]. 
The calculation leads to a number of solutions of the governing differential equations (24), each valid on a particular sub-domain as shown in figure (5). With :

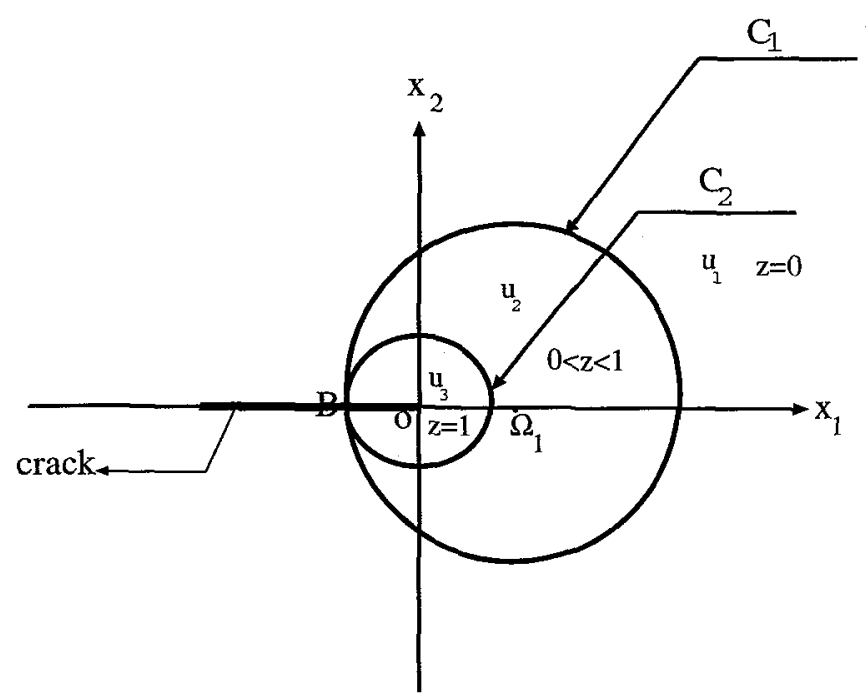

Figure 5: Domains of validity of the solutions.

$$
\begin{aligned}
u_{1} & =\frac{K_{I I I}}{\mu_{1}} \sqrt{\frac{2 r_{1}}{\pi}} \sin \frac{\theta_{1}}{2} \\
u_{2} & =\frac{K_{I I I}^{2}}{\sqrt{2} \mu_{1} \mu_{2} \pi F} \sqrt{1+\frac{x_{2}}{\sqrt{\left(x_{1}+\frac{K_{I L I}^{2}}{4 \mu_{1} \mu_{2} \pi F^{2}}\right)^{2}+x_{2}^{2}}}} \\
u_{3} & =\left(\frac{\mu_{1}}{\mu_{2}}\right)^{\frac{1}{2}} K_{I I I} \sqrt{\frac{2 r}{\pi}} \sin \frac{\theta}{2} \\
z & =\frac{|\nabla u|-E}{F-E}
\end{aligned}
$$

$C_{1}$ is the circle with center $\Omega_{1}(H, 0)\left(H=\frac{K_{L I}^{2}}{\mu_{1} \pi}\left[\frac{1}{\mathcal{A}}\left(\frac{F-E}{F \cdot E}\right)+\frac{1}{4 \mu_{2} F^{2}}-\frac{1}{4 \mu_{1} E^{2}}\right], \mathcal{A}=2 \sqrt{\frac{l \mu_{1} \mu_{2}}{\mu_{1}-\mu_{2}}}\right)$ and radius $\frac{K_{I I}^{2}}{4 \mu_{1}^{2} E^{2} \pi}$ while $C_{2}$ is the circle with center $O(0,0)$ and radius $\frac{K_{I L}^{2}}{4 \mu_{1} \mu_{2} \pi F^{2}}$. $\left(r_{1}, \theta_{1}\right)$ are the polar coordinates when the origin of the reference is changed in $\Omega_{1}(H, 0)$.

Many authors have considered the same problem, including Abeyaratne [11], for a class of incompressible, homogenous, isotropic, elastic materials whose constitutive behaviour is such that the equations of equilibrium loose ellipticity at sufficiently severe deformation. The advantage of our model is the explicit expression of the fractions of phases.

\section{Applications to shape memory alloys (pseudo-elasticity).}

Shape memory alloys display pseudo-elastic behaviour for a certain range of temperatures. No residual strains are observed upon unloading, nevertheless stress-strain curves present an hysteresis loop. This particular behaviour is caused by martensitic transformation [5]. 
In this section, the model proposed is applied to describe this hysteresis effect . Only the mechanical aspect is discussed. The temperature is assumed constant and the one-dimensional case is considered only.

Let $z$ be the fraction of martensitic phase at local strain $f$ and (1-z) the fraction of austenitic phase at local strain $e$. The phase energies are :

$$
U(e)=\frac{1}{2} K e^{2}, \quad V(f)=\frac{1}{2} K\left(f-\epsilon_{0}\right)^{2},
$$

The hysteresis loop in the stress-strain curve indicates that the phase change process is dissipative. To describe this behaviour, we choose this particular expression of the dissipation potentiel :

$$
D=|k| \dot{z} \text { when } 0<z<1
$$

which means that dissipative force must satisfy $-k \leq A \leq k, k$ is the dissipative energy per unit volume associated with phase change. The stress-strain curve obtained is presented in figure (6).

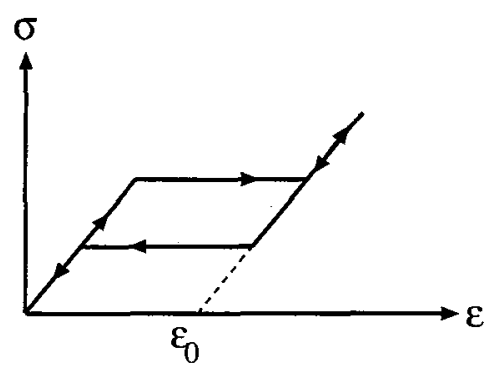

Figure 6: Hysteresis loop.

\section{Conlusion}

In this paper a simple model of solid-solid phase change was presented. The basic hypothesis consists of admetting the existence of a zone in which the phases are mixed. The three dimensional extension is easly developed [10]. This allow us to apply the model to solve boundary value problems with complex geometrie and loading. The suitable choice of the potentials (elastic energies and dissipation potential) allows the description of many different phenomena : partial brittle damage and pseudoelasticity.

\section{References}

[1] Frémond M. Matériaux à mémoire de forme, C. R. Acad. Sci. Paris, 301 série II, 1987, p. 239-244.

[2] Moreau J.J., On unilateral constraints, friction and plasticity, Cours CIME, Bressanone 1973, edition Cremonese, Roma 1974.

[3] Germain P., Cours de mécanique des milieux continus, Masson, Paris, 1973.

[5] Müller I. and Xu H., Non equilibrium thermodynamics of pseudoelasticity, Continuum Mechanics and themodynamics, 5, 3, 163-204 1993.

[6] Nguyen Q.S., Stabilité des systèmes élastiques, Cours de D.E.A, Ecole Polytechnique, 1993. 
[8] Nguyen Q.S., Bifurcation and stability in dissipative media. Applied Mechanics Review, 1994, pp $1-31$.

[9] Francford G. et Marigo J.J. Stable damage evolution in a brittle continuous medium, European Journal of Mechanics, (1993), pp 1-41.

[10] Moumni Z., Sur la modélisation de changement de phase solide : application aux matériaux à mémoire de forme et à l'endommagement fragile partiel, Thesis, E.N.P.C, 1995.

[11] Abeyaratne R., Discontinuous deformation gradients away from the tip of a crack in anti-plane shear, J. of elasticity, Vol. 11, pp :373-393, 1981. 